\title{
Using different approaches to assess the reproducibility of a culturally sensitive quantified food frequency questionnaire
}

\author{
Wentzel-Viljoen E, PhD Dietetics, $\mathrm{RD}(\mathrm{SA}$ \\ Centre of Excellence for Nutrition, North-West University, Potchefstroom \\ Laubscher R, BCom \\ Biostatistics Unit, South African Medical Research Council, Parow \\ Kruger A, PhD Nutrition \\ Africa Unit for Transdisciplinary Health Research (AUTHeR), Faculty of Health Sciences, North-West University, Potchefstroom \\ Correspondence to: Dr Edelweiss Wentzel-Viljoen, e-mail: edelweiss.wentzel-viljoen@nwu.ac.za \\ Keywords: quantified food frequency questionnaire, reproducibility
}

\section{Abstract}

Objective: To report on the use of different approaches to assess the reproducibility of a culturally sensitive quantified food frequency questionnaire (QFFQ) used for assessment of the habitual dietary intake of Setswana-speaking adults in the North West Province of South Africa.

Method: A previously developed and validated QFFQ was completed by trained fieldworkers. Portion sizes were estimated using different methods. Food intake was coded and analysed for nutrient intake per day for each subject. The first interview $(n=1888)$ took place during the baseline data collection period. For the second interview $(n=175)$, a convenient sample from the subjects who had completed the first interview was collected and the interview was conducted within four to six weeks of the first interview.

Results: There were good correlations between the first and second QFFQ for all the nutrients $(p<0.0001)$. The Wilcoxon signed-rank test showed that there were no significant differences in the median intake between the two administrations, except for energy and total fat. The Bland-Altman plots showed good agreement. Between $41 \%$ and $58 \%$ of the subjects were correctly classified into the same quartile, with less than $3 \%$ grossly misclassified. The weighted $\kappa$ statistics showed moderate agreement between the two applications.

Conclusion: Our results show that more than one statistical approach is needed to assess the reproducibility of a QFFQ. The reproducibility of this culturally sensitive QFFQ was good.

\section{Introduction}

South Africa has 11 official languages, emphasising the rich cultural and ethnic diversity of the country. The food intake of individuals is influenced by the beliefs and behaviours of the different ethnic and cultural groups and societies and by the availability of food in a specific region, resulting in differences in eating patterns, types of food consumed and nutrient content of the diet. ${ }^{1-3}$

Good-quality dietary intake data are important for the study of dietary habits and health relationships. Various dietary assessment methods, both quantitative and qualitative, are available to obtain information on dietary intake from individuals or populations, each with its own limitations, advantages and disadvantages. ${ }^{4}$ The choice of the method to use is dependent on the purpose and objectives of the study, the characteristics of the study group and available resources. For example, a single 24-hour recall may be used to calculate the average intake of a group, but one needs multiple 24-hour recalls to assess the "usual" intakes of individuals for ranking or correlation with other nutritional status variables.

The food frequency questionnaire (FFQ) is used to assess the frequency with which a food item or food group is consumed during a specified time period and provides descriptive qualitative information about usual food consumption patterns. ${ }^{4}$ By quantifying the portion size of the food item consumed, it is possible to calculate energy and nutrients and obtain information on the habitual dietary intake of an individual. The quantified food frequency questionnaire (QFFQ) can then be used to divide individuals into quartiles or quintiles of nutrient intake and to calculate the corresponding average biomarker for each quartile or quintile. The FFQ is the method of choice used by epidemiologists to assess the interrelationship between nutrient intakes of individuals and other indices of health status or disease measured in the same persons. ${ }^{4}$

"Validity" refers to the degree to which a dietary method measures what it is supposed to measure, while "reproducibility" means that the method gives very similar results when used repetitively in similar situations. ${ }^{4}$ Gibson argued that true reproducibility is not possible but that only an estimate of reproducibility can be made since exact duplication of an FFQ is not possible. ${ }^{4} \mathrm{FFQs}$ are developed to be sensitive to the dietary intake of different cultures and regions, ${ }^{5-7}$ and should be validated and tested for reproducibility to be of value. ${ }^{4}$

The Prospective Urban and Rural Epidemiological (PURE) study is a prospective cohort study tracking changing lifestyles, risk factors and chronic disease in urban and rural areas of 13 countries in 
transition. The South African part (PURE-SA) was conducted in two areas of the North West Province, a province typically in transition.

A culturally sensitive QFFQ had been previously developed ${ }^{5}$ and validated $^{8}$ for use in an African population. The objective of this paper is to report on the reproducibility of this culturally sensitive QFFQ used for assessment of the habitual dietary intake of Setswanaspeaking adults in the North West Province of South Africa who participated in the PURE-SA study.

\section{Method}

\section{Research setting and study population}

Both a rural and an urban area were identified for the study. The rural area included Ganeysa, $70 \mathrm{~km}$ from the Botswana border, and two deep-rural communities, Moswane and Tlakgameng, $35 \mathrm{~km}$ east from Ganyesa. Moswane and Tlakgameng are still under tribal law. The urban area included lkageng, a township next to Potchefstroom, as well as Zonderwater extensions 7 and 11, and Top City. Extensions 7 and 11 and Top City are informal settlements surrounding Ikageng. A total of 1006 rural and 1003 urban apparently healthy men and women, randomly selected and between 35 years and 70 years of age, participated voluntarily in the bigger PURE-SA study.

\section{Quantified food frequency questionnaire}

The previously developed ${ }^{5}$ and validated ${ }^{8}$ culturally sensitive QFFQ was completed by trained fieldworkers in the respondents' language of choice. Sound knowledge of the food habits of the study population was obtained using both quantitative and qualitative methods in the form of in-depth individual interviews and focus group discussions. ${ }^{5}$ The QFFQ was tested for relative validity, using seven-day weighed records and biomarkers in the Transition and Health during Urbanisation of South Africans (THUSA) study, and it appeared to be able to measure the intake of staple and frequently consumed foods satisfactorily in this population. ${ }^{8}$ The PURE-SA subjects were of the same cultural group and demographic region as the THUSA study population and therefore the same QFFQ was used.

For the present PURE-SA study, portion sizes were estimated using food portion photographs, ${ }^{9}$ appropriate utensils and containers, and examples of specific foods. Portion sizes were reported in household measures and were converted to weights using standard tables. ${ }^{10}$ The food intake was coded according to the South African food composition database of the South African Medical Research Council (MRC). ${ }^{11-13}$ The nutrient content of food items consumed by this population, but who were not on the database, was either analysed or sourced from other food composition databases [i.e. United States Department of Agriculture (USDA) National Nutrient Database Release 23], added to the database and coded.

For example, the nutrient content of gemaaldes, a mixture of beef lung and beef fat, was sampled according to standard procedures and analysed for total fat and fatty acid composition by the MRC. An assumption was made that the nutrient content of donkey meat is more or less equal to that of horse meat, and this was sourced from the USDA National Nutrient Database Release 23. The questionnaire was completed for food items eaten over the previous 30 days. The dietitian responsible for the training of the fieldworkers checked the completed QFFQs, in the presence of the fieldworker, on a weekly basis. If a $Q F F Q$ was incomplete or the dietitian had any queries, the fieldworker was requested to return to the household to complete or correct the QFFQ. The grams of food intake were calculated for seven days to be able to include quantities less than one gram per day in the analysis. Thereafter the nutrient content was calculated and expressed as average nutrient amounts consumed per day for each subject. To test reproducibility, only energy, the macronutrients, dietary fibre, three minerals and six vitamins were used.

\section{Study sample}

A QFFQ was completed during the baseline data collection period of the bigger PURE-SA study. This interview was also used as the first completed QFFQ (QFFQ1). The second interview (QFFQ2) was conducted on a convenient sample of 175 subjects from the bigger group of subjects within four to six weeks of the first interview. Both men and women and subjects from both the rural and urban area were interviewed. The same procedures as for the first interview were followed and an equal number of days of the week were covered.

\section{Fieldworkers}

With the help and support of the local Department of Health and community leaders, eight Setswana-speaking fieldworkers who had completed their secondary school education were selected from each community (eight living in the urban and eight living in the rural communities). They were extensively trained as fieldworkers in research methodology. A dietitian, experienced in dietary methodology and the training of fieldworkers trained the fieldworkers over a period of three days to complete the QFFQ and to quantify the amount of food eaten. A training manual was developed for this purpose. Food models, food pictures, real food, food dishes and different utensils, such as teaspoons, tablespoons, ladles and cups, were used during the training to standardise the completion of the QFFQ and to assess quantities. Both questionnaires (QFFQ1 and QFFQ2) were completed in the house of the subject in Setswana. The same fieldworkers completed QFFQ1 and QFFQ2.

\section{Statistical analysis}

As a result of the skewness of the data, nonparametric methods were used in the analysis. Spearman correlation coefficients were calculated to determine the strength of the relationship between the nutrient intakes reported at the first and second interviews. The Wilcoxon matched-pairs signed-rank test was used to assess agreement between median nutrient intakes on a group basis. The Bland and Altman ${ }^{14}$ method was used to evaluate agreement between the two interviews for energy and the macronutrients. The mean of QFFQ1 and QFFQ2 was plotted against the difference between the two applications (QFFQ2-QFFQ1). Assessing agreement was also done by classifying subjects into four categories (quartiles) of intake by QFFQ1 and QFFQ2 and calculating the percentage of subjects correctly classified into the same category and those grossly misclassified.

Misclassification error was assessed using the weighted $\kappa$ statistic. Weighted $\kappa$ is valuable in that it gives a single value to represent agreement and adjusts for chance agreement and the degree of disagreement. ${ }^{15} \mathrm{~A} \mathrm{p}$-value of less than 0.05 was used to indicate statistical significance. 


\section{Ethical considerations}

The PURE-SA study protocol complied with the Helsinki Declaration, as revised in 2000, and was approved by the Ethics Committee of the North-West University, Potchefstroom Campus (No. 04M10). Permission for the study to be conducted was also obtained from the Provincial Department of Health of the North West Province and the local government authorities of each town as well as the tribal chief in the rural communities. Before enrolment in the study as well as with every follow-up action, all volunteers gave informed written consent.

\section{Results}

More women (60.6\%) than men (39.4\%) participated in this study and more subjects resided in the urban area $(52.6 \%)$ than in the rural area $(47.4 \%)$. The majority of the women (26\%) and men (23\%) were between 45 and 49 years old.

Table I gives the mean, standard deviation and median intake for energy, the macronutrients and some minerals and vitamins, and the Spearman correlation coefficients. There were good correlations between the first and second completed QFFQs for all the nutrients, with the correlation coefficient ranging from 0.57 for vitamin $A$ to 0.76 for protein. All these correlations were statistically significant $(p<0.001)$. The median of alcohol intake on both occasions shows that $50 \%$ of the subjects did not consume any alcohol.

Table II shows that there were no significant differences in the median intake between the two administrations of the QFFQ, except for energy and total fat. Further analysis of this difference showed that there was a significant difference for energy and total fat in the urban group but not in the rural group (data not shown).
Table II: Median nutrient intake and Wilcoxon signed-rank test assessing agreement between median nutrient intakes on a group basis between the two administrations for a selection of nutrients $(n=175)$

\begin{tabular}{|l|c|c|c|}
\hline \multirow{2}{*}{ Nutrient } & QFFQ1 $(\mathbf{n}=\mathbf{1 7 5})$ & QFFQ2 $(\mathbf{n = 1 7 5 )}$ & \multirow{2}{*}{ p-value } \\
\cline { 2 - 3 } & Median & Median & \\
\hline Energy $(\mathrm{kJ})$ & 6970.0 & 7385.0 & $0.0028^{*}$ \\
\hline Total protein $(\mathrm{g})$ & 48.9 & 48.1 & 0.0546 \\
\hline Total fat $(\mathrm{g})$ & 39.4 & 43.3 & $0.0003^{\star}$ \\
\hline Alcohol $(\mathrm{g})$ & 0 & 0 & 0.9205 \\
\hline Total carbohydrate $(\mathrm{g})$ & 256.9 & 257.7 & 0.0067 \\
\hline Dietary fibre $(\mathrm{g})$ & 18.5 & 18.3 & 0.2722 \\
\hline Calcium $(\mathrm{mg})$ & 271.1 & 299.0 & 0.0507 \\
\hline Iron $(\mathrm{mg})$ & 11.0 & 11.8 & 0.0992 \\
\hline Zinc $(\mathrm{mg})$ & 8.5 & 8.9 & 0.1559 \\
\hline Vitamin A $(\mathrm{RE})$ & 603.5 & 674.4 & 0.8401 \\
\hline Thiamine $(\mathrm{mg})$ & 1.4 & 1.4 & 0.6201 \\
\hline Riboflavin $(\mathrm{mg})$ & 1.0 & 1.1 & 0.1383 \\
\hline Niacin $(\mathrm{mg})$ & 12.8 & 12.9 & 0.1300 \\
\hline Folate $(\mu \mathrm{g})$ & 350.9 & 350.4 & 0.9124 \\
\hline Vitamin $\mathrm{B}_{12}(\mu \mathrm{g})$ & 3.0 & 3.1 & 0.2454 \\
\hline
\end{tabular}

${ }^{*} \mathrm{p}<0.05$ (indicates statistical significance)

Figures 1 to 4 show the Bland-Altman plots for energy and the macronutrients, including the $95 \%$ confidence interval $(\mathrm{Cl})$. These plots reveal the relationship between the difference between the first and second administration of the QFFQ (QFFQ2 and QFFQ1) and the mean of the two administrations.

Table I: Mean, standard deviation and median nutrient intake and Spearman rank correlation coefficients between the two administrations for a selection of nutrients $(\mathrm{n}=175)$

\begin{tabular}{|c|c|c|c|c|c|c|c|}
\hline \multirow{2}{*}{ Nutrient } & \multicolumn{3}{|c|}{ QFFQ1 $(n=175)$} & \multicolumn{3}{|c|}{ QFFQ2 ( $n=175)$} & \multirow[b]{2}{*}{$\mathbf{r}$} \\
\hline & Mean & SD & Median & Mean & SD & Median & \\
\hline Energy (kJ) & 7448.0 & 3209.0 & 6970.0 & 8198.0 & 3871.0 & 7385.0 & $0.74^{*}$ \\
\hline Total protein (g) & 53.6 & 25.1 & 48.9 & 58.1 & 31.0 & 48.1 & $0.76^{*}$ \\
\hline Total fat $(\mathrm{g})$ & 45.8 & 27.5 & 39.4 & 53.5 & 35.8 & 43.3 & $0.75^{\star}$ \\
\hline Total carbohydrate $(\mathrm{g})$ & 261.6 & 105.8 & 256.9 & 283.2 & 123.0 & 257.7 & $0.71^{*}$ \\
\hline Dietary fibre $(\mathrm{g})$ & 20.0 & 8.7 & 18.5 & 20.9 & 10.4 & 18.3 & $0.69^{*}$ \\
\hline Calcium (mg) & 327.3 & 219.7 & 271.1 & 360.6 & 256.4 & 299.0 & $0.64^{*}$ \\
\hline Iron (mg) & 12.6 & 5.7 & 11.0 & 13.5 & 6.6 & 11.8 & $0.70^{*}$ \\
\hline Zinc (mg) & 9.8 & 4.6 & 8.5 & 10.4 & 5.3 & 8.9 & $0.74^{\star}$ \\
\hline Vitamin A (RE) & 831.7 & 623.3 & 603.5 & 869.7 & 662.0 & 674.4 & $0.57^{*}$ \\
\hline Thiamine (mg) & 1.6 & 0.7 & 1.4 & 1.7 & 0.8 & 1.4 & $0.69^{*}$ \\
\hline Riboflavin (mg) & 1.2 & 0.6 & 1.0 & 1.3 & 0.8 & 1.1 & $0.72^{*}$ \\
\hline Niacin (mg) & 13.9 & 6.6 & 12.8 & 15.1 & 8.4 & 12.9 & $0.73^{*}$ \\
\hline Folate $(\mu \mathrm{g})$ & 379.0 & 163.8 & 350.9 & 385.7 & 197.3 & 350.4 & $0.66^{\star}$ \\
\hline Vitamin $B_{12}(\mu \mathrm{g})$ & 4.2 & 4.1 & 3.0 & 5.0 & 5.4 & 3.1 & $0.59^{*}$ \\
\hline
\end{tabular}

${ }^{\star} \mathrm{p}<0.0001$ (indicates statistical significance) 


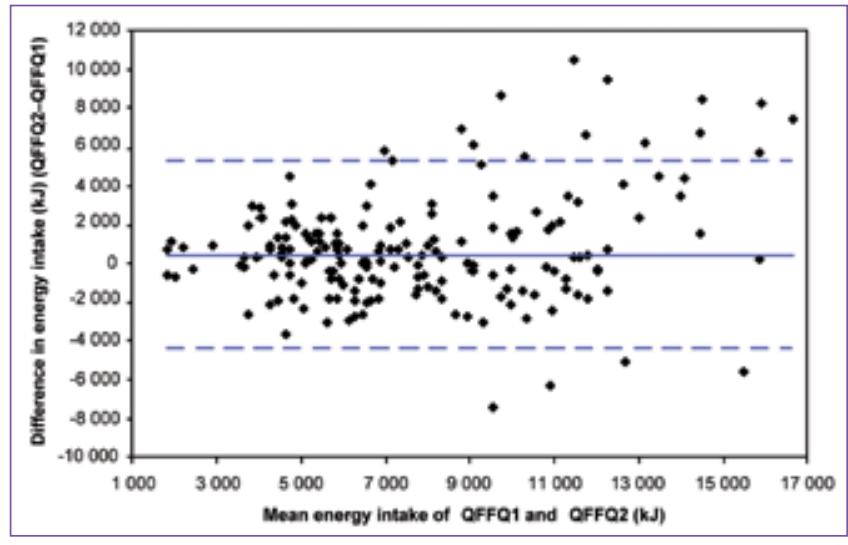

Figure 1: Bland-Altman plot for energy $(n=175)$ with the mean difference (solid line) and $95 \%$ limits of agreement (broken lines)

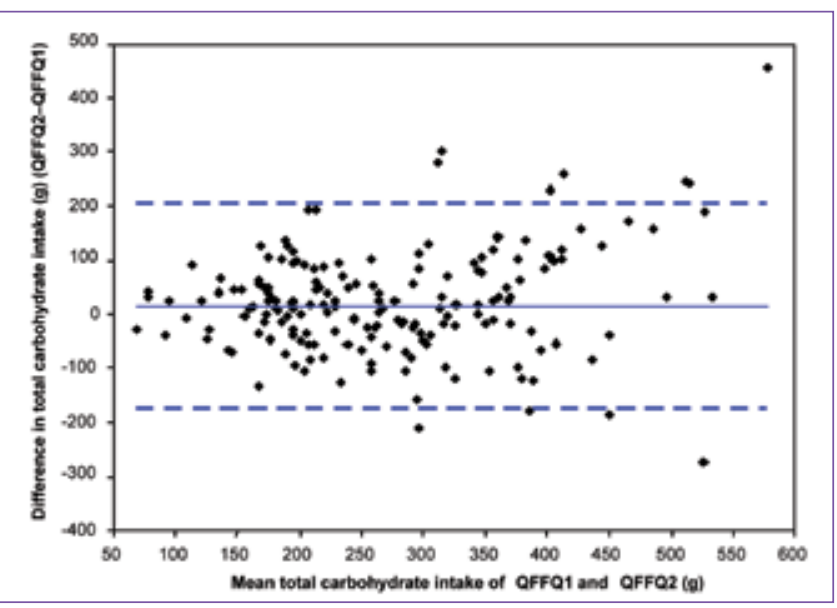

Figure 3: Bland-Altman plot for total carbohydrate $(n=175)$ with the mean difference (solid line) and $95 \%$ limits of agreement (broken lines)

In Figure 1, the Bland-Altman plot for energy, expressed in kilojoules, shows good agreement, but the QFFQ2 tends to overestimate with higher average energy intake. The mean difference in energy intake tends to cluster around the zero line. Only 14 subjects fall above the upper limit of agreement and only four fall below the lower limit of agreement.

Figure 2 shows the Bland-Altman plot for protein. The plot is scattered and the mean difference in total protein intake is equal to the zero line and tends to cluster around the zero line. With an increase in intake, QFFQ2 tends to overestimate intake. More subjects fall above the upper limit of agreement than below the lower limit of agreement.

Figure 3 shows the Bland-Altman plot for carbohydrates. The plot shows a fairly good agreement with a bias toward overestimation in QFFQ2. At higher mean intakes (difference between QFFQ2 and QFFQ1) of about $300 \mathrm{~g}$, both QFFQ1 and QFFQ2 tend to overestimate intake. Very few subjects fall above the upper or lower limit of agreement.

The Bland-Altman plot for total fat intake (Figure 4) exhibits clustering around the mean difference in total fat intake up to about $40 \mathrm{~g}$ indicating a consistent bias, but with higher average intakes both applications tend to overestimate intake. Few subjects fall above and below the upper and lower limit of agreement.

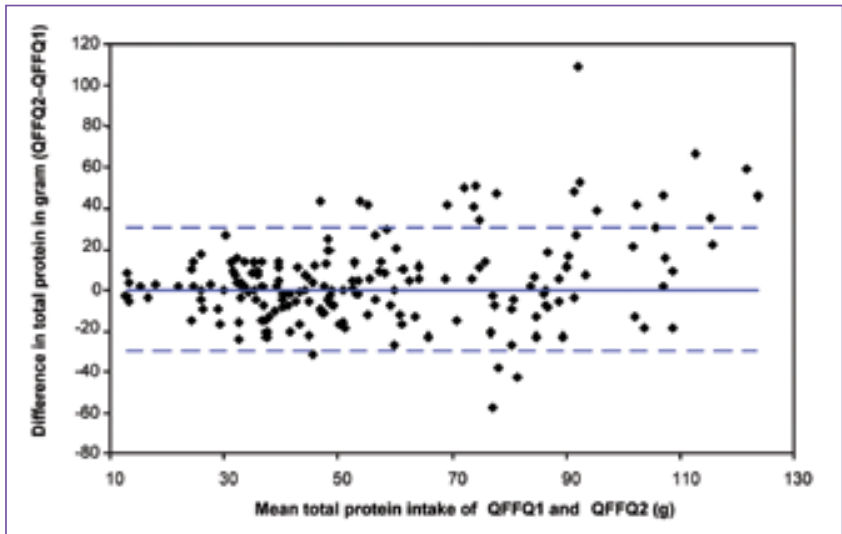

Figure 2: Bland-Altman plot for total protein $(n=175)$ with the mean difference (solid line) and $95 \%$ limits of agreement (broken lines)

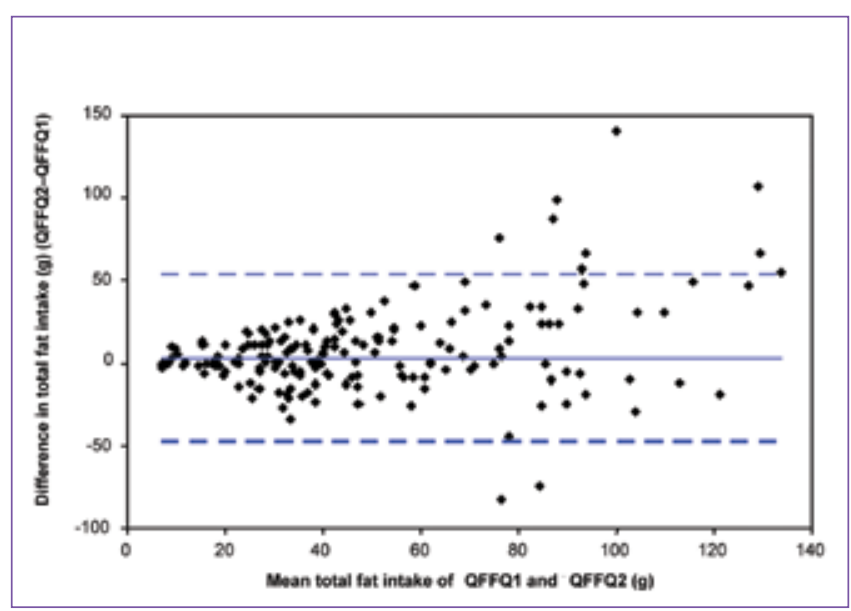

Figure 4: Bland-Altman plot for total fat $(n=175)$ with the mean difference (solid line) and 95\% limits of agreement (broken lines)

Table III shows whether a subject was placed into the same category on intake by QFFQ1 and QFFQ2 or grossly misclassified. The error of misclassification was assessed using the weighted $\kappa$ statistic. More than $50 \%$ of the subjects were classified into the same quartile for energy, total protein and total fat. Nearly $50 \%$ of the subjects were classified into the same quartile for total carbohydrate. The number of subjects classified into the same quartile was the lowest for vitamin $A(41.1 \%)$ and vitamin $B_{12}(41.1 \%)$. No subject was grossly misclassified for energy, total protein, total fat and niacin. For total carbohydrate, only two subjects were grossly misclassified. The highest number of subjects grossly misclassified was for vitamin $A$ and vitamin $B_{12}$, but only four participants of the 175 for each nutrient. The $\kappa$ statistics for all the nutrients were between 0.41 and 0.59 .

\section{Discussion}

The QQF has been recommended as the primary method for assessing dietary intake in epidemiological studies to establish the role of diet on disease outcome. ${ }^{16}$ This paper reports on the reproducibility of a previously validated $Q F F Q$ to estimate the habitual nutrient intake of a Setswana-speaking population in the North West Province of South Africa.

It has been suggested that more than one analytical approach should be used to assess the validity and reproducibility of dietary 
Table III: Percentage of subjects classified into the same quartile and grossly misclassified on both occasions, and weighted kappa ( $\mathrm{KW})$

\begin{tabular}{|l|c|c|c|}
\hline Nutrient & $\begin{array}{c}\text { \% correctly classified } \\
\text { into the same quartile } \\
\mathbf{n = 1 7 5}\end{array}$ & $\begin{array}{c}\text { \% grossly } \\
\text { misclassified } \\
\mathbf{n}=175\end{array}$ & $\kappa_{\mathrm{w}}$ \\
\hline Energy & 50.3 & 0.0 & $0.52^{\star}$ \\
\hline Total protein & 58.3 & 0.0 & $0.59^{\star}$ \\
\hline Total fat & 55.4 & 0.0 & $0.57^{\star}$ \\
\hline Dietary fibre & 46.9 & 0.6 & $0.46^{\star}$ \\
\hline Total carbohydrate & 49.7 & 1.1 & $0.49^{\star}$ \\
\hline Calcium & 51.4 & 1.7 & $0.46^{\star}$ \\
\hline Iron & 54.3 & 1.1 & $0.54^{\star}$ \\
\hline Zinc & 56.0 & 0.6 & $0.57^{\star}$ \\
\hline Vitamin A & 41.1 & 2.3 & $0.41^{*}$ \\
\hline Thiamine & 54.9 & 1.1 & $0.54^{*}$ \\
\hline Riboflavin & 53.7 & 1.7 & $0.52^{\star}$ \\
\hline Niacin & 54.3 & 0.0 & $0.57^{\star}$ \\
\hline Folate & 51.4 & 1.1 & $0.48^{\star}$ \\
\hline Vitamin $\mathrm{B}_{12}$ & 41.1 & 2.3 & $0.41^{\star}$ \\
\hline
\end{tabular}

${ }^{*}$ Moderate agreement for all nutrients (interpretation based on Masson et al ${ }^{15}$ )

assessment tools. ${ }^{1,8,9,17}$ Four different methods were used in this study: firstly, the Spearman correlation coefficients were used to determine the strength of the relationship between the nutrient intakes reported at the first and second interviews; secondly, the Wilcoxon matched-pairs signed-rank test was used to assess the agreement of the nutrient intakes; thirdly, the Bland and Altman ${ }^{8}$ method was used to further evaluate the limits of agreement between the two interviews for energy and the macronutrients; and fourthly, assessing agreement was also done by classifying subjects into four categories (quartiles) of intake by QFFQ1 and QFFQ2 and calculating the percentage of subjects correctly classified into the same category and grossly misclassified.

The first interview was conducted as part of the baseline study of PURE-SA. The second interview, to complete the QFFQ, was done within four to six weeks of the first interview and in 175 participants, using a convenient sample from the baseline study. This is within the recommendations that the second interview should take place within four to eight weeks ${ }^{18}$ of the first interview and that between 100 and 200 subjects ${ }^{19}$ should participate in this type of study.

The correlations for energy, macronutrients, mineral intake and vitamin intake between the two applications were good and higher (between 0.59 and 0.76 ) than the threshold of 0.4 suggested by Cade et $\mathrm{al}^{19}$ and above the 0.5 threshold suggested by Masson et $\mathrm{al}^{15}$ for all the nutrients. All the correlations were statistically significant. Macintyre et al, ${ }^{5}$ who conducted a reproducibility study with the same QFFQ in Setswana-speaking subjects in the North West province, reported far lower correlations, ranging from 0.14 for calcium to 0.39 for fat. All these correlations were lower than the threshold of 0.4 suggested by Cade and colleagues, ${ }^{19}$ with the exception of alcohol $(r=0.75)$. Similar correlations as in the present study were found in the NORBAGREEN ${ }^{20}$ study and in a study of Chinese women, ${ }^{21}$ while lower ${ }^{22,23}$ and higher ${ }^{24}$ correlations were reported in other studies.
In a reproducibility study done in Japan, using an interval period from three days to five years, similar median correlation coefficients were reported (0.50-0.72), with intervals between nine months and one year, but this dropped to 0.24 if the second administration was done five years after the first. ${ }^{25} \mathrm{~A}$ longer time lapse of 2.15 years (range 1.65-2.66) between the first and second administration of the food frequency questionnaire was also used in the Shanghai Women's Health Study ${ }^{26}$ The authors concluded that the reproducibility of the FFQ is likely to be underestimated in their study, explaining that the decrease in the correlation was caused by the change in dietary intake during the two years since nutrient and food intake from the second FFQ was lower than the first due to ageing.

Satisfactory agreement between the intakes for QFFQ1 and QFFQ2 was demonstrated by the nonsignificant difference between the group median intakes for the two sets of data, with the exception of energy and total fat. Both energy and fat intake were higher with the second administration of the QFFQ. However, the Wilcoxon signedrank test does not take into account the confounding effect of withinsubject variation on usual nutrient intakes. ${ }^{4}$ MacIntyre et al. ${ }^{5}$ found that the mean (not median) intakes of the second administration of the QFFQ were higher than the first application, with the exception of carbohydrate and alcohol, and that the differences were not significant for six of the nutrients tested.

The level of agreement between the two administrations is depicted in the Bland-Altman plots. They show across the range of intakes that the agreement does not seem to be different at either higher or lower levels of consumption and thus may not lead to differential misclassification of exposure and thus biased estimates of effect on outcomes. MacIntyre et al ${ }^{5}$ also reported good agreement for energy, protein, carbohydrate and fat in their study using the same QFFQ and in a similar population group.

The last approach to assess agreement between the two administrations of the QFFQ entailed classifying subjects into four categories (quartiles) of intake. To test whether the QFFQ was able to place a subject into the same subgroup of intake for both administrations, the percentage of subjects correctly classified into the same category and grossly misclassified were calculated. Between $41 \%$ and $58 \%$ of the subjects were correctly classified into the same quartile, with less than $3 \%$ grossly misclassified with all the nutrients, demonstrating moderate agreement (weighted kappa, $\kappa_{w}$, between $0.41-0.60$ ). Weighted kappa values above 0.4 are desirable for nutrients of interest if the possibility of false-negative associations between diet and disease in epidemiology studies is to be minimised. ${ }^{15}$ These results indicate that this QFFQ can be used for categorising individuals according to levels of intake, which is usually done in epidemiological studies.

Macintryre et al, ${ }^{5}$ who conducted a reproducibility study using the same QFFQ in a similar population group, classified $84 \%$ and $75 \%$ of the subjects in the same quintile for vitamin $\mathrm{C}$ and alcohol respectively. For energy, only $22 \%$ were classified into the same quintile, while we classified $50 \%$ into the same quartile. In a reproducibility study conducted in Spain, the authors reported gross misclassification of nutrients between $1.5 \%$ (e.g. carbohydrate and total fat) and $6.1 \%$ (mono-unsaturated fatty acids). ${ }^{27} \mathrm{~A}$ study from the Australian Cancer and Ovarian Cancer Study Groups reported the reproducibility of a 
semi-quantitative FFQ in 100 adults and found slightly higher $\kappa_{w}$ than the present study, ranging between 0.44 (starch) and 0.83 (alcohol). ${ }^{28}$ The $\kappa_{\mathrm{w}}$ was similar for the energy and the macronutrients between the two studies. The reliability of an FFQ in adolescents in Vietnam was assessed for short-term (four-week interval) and longterm (six-month interval) reliability. ${ }^{29}$ For the short-term reliability, the percentage of subjects grossly misclassified was higher than in the present study, with protein $5.5 \%$ and iron $3.2 \%$ in the Vietnam study. ${ }^{29}$ The $\kappa_{w}$ was above 0.4 for all the nutrients, showing fair to good agreement. ${ }^{29}$

Reproducibility could have been influenced by the fact that the questionnaires were completed by trained fieldworkers and not by the participants themselves. In this study, the same fieldworker conducted the interview on both occasions. However, Teufel recommended that the two administrations be conducted by two different interviewers, as it would help to reduce the need of the respondent to recall earlier responses by eliminating the feeling that the same interviewer had come to check on earlier responses and would therefore assist in determining the effectiveness of the instrument. ${ }^{30}$ However, in the present study the between- and within-interviewer reproducibility was not tested.

\section{Conclusion}

While the relationship between the first and second application of the QFFQ was good, as indicated by the Spearman correlation coefficients, there were significant differences in the median for energy and fat between QFFQ1 and QFFQ2 (Wilcoxon signed-rank test). The level of agreement between the two administrations, as indicated by the Bland-Altman plots, did not seem to be different at different levels of consumption, and the $\kappa_{w}$ also demonstrated moderate agreement. These results show that more than one statistical approach is needed to assess the reproducibility of a QFFQ. The PURE-SA study relies on this QFFQ for reliable information on habitual food and nutrient intake to understand and explain dietrelated disease risk.

In summary, the reproducibility of this culturally sensitive QFFQ was good and suggests that it could be used for classifying individuals into groups based on their habitual dietary intake. It is recommended that this QFFQ be used in other groups with similar eating habits as the Setswana culture and probably in age groups younger than 35 years, provided that the subjects can recall dietary intake over a period of one month.

\section{Acknowledgements}

The authors would like to thank all supporting staff and the participants of the PURE study, and in particular the following: PURE-South Africa: the PURE-SA research team, fieldworkers and office staff in the Africa Unit for Transdisciplinary Health Research (AUTHeR), Faculty of Health Sciences, North-West University, Potchefstroom, South Africa; PURE International: Dr S Yusuf and the PURE project office staff at the Population Health Research Institute (PHRI), Hamilton Health Sciences and McMaster University, ON, Canada; and the organisations providing funding.

\section{Declarations}

This research received funding from the South Africa-Netherlands Research Programme on Alternatives in Development (SANPAD), the South African National Research Foundation (NRF GUN Nos. 2069139 and FA2006040700010), North-West University, the Public Health Research Institute and the South African Medical Research Council.

\section{References}

1. Steyn NP, Bradshaw D, Norman R, et al. Dietary changes and the health transition in South Africa: implications for health policy. Cape Town: South African Medical Research Council; 2006.

2. Wolmarans $\mathrm{P}$, Seedat YK, Mayet FGH, et al. Dietary intake of Indians living in the metropolitan area of Durban. Public Health Nutr. 1999;2:50-60.

3. Mia FB, Vorster HH. Coronary heart disease risk factors in Indian adolescents - the role of diet. Cardiovasc J S Afr. 2000;11:68-75.

4. Gibson RS. Principles of nutritional assessment. $2^{\text {nd }}$ edition. Dunedin, New Zealand: Oxford University Press; 2005.

5. MacIntyre UE, Venter CS, Vorster HH. A cultural-sensitive quantified food frequency questionnaire used in an African population: 1. Development and reproducibility. Public Health Nutr. 2000;4:53-62.

6. Merchant AT, Dehghan M, Chifamba J, et al. Nutrient estimation from an FFQ developed for a black Zimbabwean population. Nutr J. 2005;4:37.

7. Iqbal R, Ajayan $\mathrm{K}$, Bharathi AV, et al. Refinement and validation of an $\mathrm{FFQ}$ developed to estimate macroand micronutrient intakes in a south Indian population. Public Health Nutr. 2008;12:12-18.

8. MacIntyre UE, Venter CS, Vorster HH. A cultural-sensitive quantified food frequency questionnaire used in an African population: 2. Relative validation by 7-day weighed food records and biomarkers. Public Health Nutr. 2000;4:63-71

9. Venter CS, Maclntyre UE, Vorster HH. The development and testing of a food portion photograph book for use in an African population. J Hum Nutr Diet. 2000;13:205-218.

10. Langenhoven ML, Conradie PJ, Wolmarans P, Faber M. MRC food quantities manual. $2^{\text {nd }}$ edition. Parow Valley: Medical Research Council; 1991.

11. Langenhoven, ML, Kruger, M, Gouws, E, Faber, M. MRC Food Composition Tables. $3^{\text {rd }}$ edition. Parow Valley: Medical Research Council; 1991.

12. Kruger M, Sayed N, Langenhoven ML, Holing F. Composition of South African foods: vegetables and fruit. Supplement to the MRC Food Composition Tables 1991. Parow Valley: Medical Research Council; 1998.

13. Sayed N, Frans Y, Schönfeldt HC. Composition of South African foods: milk and milk products, eggs, meat and meat products. Supplement to the MRC Food Composition Tables 1991. Parow Valley: Medical Research Council; 1999.

14. Bland JM, Altman DG. Statistical methods for assessing agreement between two methods of clinical measurement. Lancet. 1986;1:307-310.

15. Masson LF, McNeill G, Tomany J0, et al. Statistical approaches for assessing the relative validity of a food-frequency questionnaire: use of correlation coefficients and the kappa statistic. Public Health Nutr. 2003;63:313-321.

16. Willett WC. Future directions in the development of food-frequency questionnaires. Am J Clin Nutr. 1994;59(Suppl):171S-174S.

17. Cade J, Thompson R, Burley V, Warm D. Development, validation and utilisation of food-frequency questionnaires - a review. Public Health Nutr. 2002;5:567-587.

18. Block G. Hartman AM. Issues in reproducibility and validity of dietary studies. Am J Clin Nutr. 1989;50:1133-1138.

19. Willet WC. Nutrition epidemiology. New York: Oxford University Press; 1998.

20. Petkeviciene J, Similä M, Becker W, et al. Validity and reproducibility of the NORBAGREEN food frequency questionnaire. Eur J Clin Nutr. 2009;63:141-149.

21. Zhang $\mathrm{CX}$, Ho SC. Validity and reproducibility of a food frequency questionnaire among Chinese women in Guangdong Province. Asia Pac J Clin Nutr. 2009;18:240-250.

22. Ahn $Y$, Kwon E, Shim JE, et al. Validation and reproducibility of food frequency questionnaire for Korean genome epidemiologic study. Eur J Clin Nutr. 2007;61:1435-1441.

23. Bae YJ, Choi HY, Sung MK, et al. Validity and reproducibility of a food frequency questionnaire to assess dietary nutrients for prevention and management of metabolic syndrome in Korea. Nutr Res Pract. 2010;4:121-127.

24. Bohlscieid-Thomas S, Hoting I, Boeing H, Wahrendorf J. Reproducibility and relative validity of energy and macronutrient intake of a food frequency questionnaire developed for the German part of the EPIC project. Int J Epid. 1997;26(Suppl 1):S71-S81.

25. Wakai K. A review of food frequency questionnaires developed and validated in Japan. J Epidemiol. 2009;19:1-11

26. Shu $\mathrm{X}$, Yang $\mathrm{G}$, Jin $\mathrm{F}$, et al. Validity and reproducibility of the food frequency questionnaire used in the Shanghai Women's Health Study. Eur J Clin Nutr. 2004;58:17-23.

27. De la Fuente-Arrillaga C, Ruiz ZV, Bes-Rastrollo M, et al. Reproducibility of an FFQ validated in Spain. Public Health Nutr. 2010;13:1364-1372.

28. Ibiebele T, Parekh S, Mallitt K, et al. Reproducibility of food and nutrient intake estimates using a semiquantitative FFQ in Australian adults. Public Health Nutr. 2009;12:2359-2365.

29. Hong TK, Dibley MJ, Sibbritt D. Validity and reliability of an FFQ for use with adolescents in Ho Chi Minh City, Vietnam. Public Health Nutr. 2010;13:369-375.

30. Teufel NI. Development of culturally competent food-frequency questionnaires. Am J Clin Nutr. 1997;65(Suppl 1):173S-1178S. 